\title{
Tuneable Thiol-Ene Photo-Crosslinked Chitosan-Based Hydrogels for Biomedical Applications
}

\author{
Sarah E.S Michel ${ }^{\dagger}$, Sarah E. Rogers ${ }^{\S}$, Wuge H. Briscoe ${ }^{\dagger *}$, M. Carmen Galan ${ }^{\dagger *}$ \\ † School of Chemistry, University of Bristol, Cantock's Close, Bristol BS8 1TS, UK \\ $\S$ ISIS Neutron and Muon Source, Science and Technology Facilities Council, Rutherford \\ Appleton Laboratory, Didcot, OX11 0QX, UK
}

\begin{abstract}
Access to biocompatible hydrogels with tuneable properties is of great interest. Here we report the practical synthesis and characterisation of a series of photo-crosslinked chitosan hydrogels from norbornene-functionalised chitosan (CS-nb) and various thiolated crosslinkers. The resulting materials were characterised by NMR, swelling ratio, rheology, SEM and SANS. The new hydrogels exhibit $\mathrm{pH}$ - and salt-dependent swelling whilst the macro- and microscale properties can be modulated by the choice and degree of crosslinker or the polymer concentration. The materials can be molded in situ and loaded with small molecules that are released overtime. Moreover, the incorporation of collagen in the hydrogels drastically improves cell adhesion and excellent cell viabilities were observed with human dermofibroblast cells for up to 6 days, highlighting the potential use of these materials in the biomedical area.
\end{abstract}

Hydrogels are defined as physically or chemically crosslinked hydrophilic polymer chains swollen in water. Owing to their swollen 3D structure, they constitute an excellent mimic of human tissues and provide an appropriate scaffold for cells to grow on and have been therefore highly exploited in the fields of wound healing and tissue engineering. ${ }^{1-3}$

A wide range of natural as well as synthetic and hybrid polymers have been used to produce hydrogels via chemical or physical crosslinking. In this context, polysaccharides are highly attractive hydrogel precursors for biomedical applications as they are cheap and abundant yet biocompatible, biodegradable and bioactive materials owing to the similarities with glycosaminoglycans (GAG) and glycoproteins found on the extracellular matrix (ECM). Moreover, polysaccharides contain multiple functional groups of different reactivities enabling their tailored functionalisation and crosslinking to the need. ${ }^{4-6}$ Chitosan (CS) is the only cationic natural polysaccharide consisting of $\beta$-1,4-D-glucosamine (GlcNAc) and $\beta-1,4-\mathrm{N}$ - 
acetyl-D-glucosamine (GlcNAc) units. CS is generally obtained by deacetylation of at least 50 $\%$ of GlcNAc residues of chitin. ${ }^{7}$ The presence of free amines imparts CS with mucoadhesion properties, hemostatic action and antibacterial activity, while its similarities with GAGs provide a favourable environment for cell adhesion or proliferation. ${ }^{8-11} \mathrm{CS}$ has shown particularly promising applications in wound healing and tissue engineering as CS-based materials allow for efficient delivery of fragile bioactives such as growth factors or proteins. ${ }^{6}$, 8, 10, 12 In addition to wound healing applications, CS alone enhances vascularisation and reduces scaring. It was also shown to support attachment and proliferation of various cell lines such as fibroblasts, nerve cells or chondrocytes. ${ }^{13,14}$

Chemically photo-crosslinked CS-based hydrogels provide an extra spatio-temporal control over hydrogel formation through the position of the light source. CS photocrosslinkable precursors are typically obtained by (meth)acrylation, leading to random gel network formation. ${ }^{15}$ Alternatively, the thiol-ene photo-click reaction using norbornene (nb) as the bicyclic strained alkene partner has many advantages since nb is highly stable under physiological conditions, yet highly reactive towards radicals driven by the strain release from an $\mathrm{sp}^{2}$ alkene to an $\mathrm{sp}^{3}$ alkane. ${ }^{16,17}$ The coupling reaction proceeds with fast kinetics with various long-wavelength UV (Irgacure $2959^{18}$ - IRG) or visible-light activated photoinitiators $\left(\right.$ Eosin $\mathrm{Y}^{19}$ or riboflavin ${ }^{20}$ ).

We previously reported the synthesis of novel biocompatible CS-based microgels obtained through thiol-ene photockick crosslinking of CS-hydrogel precursors bearing a norbornene moiety (CS-nb, Figure 1). ${ }^{21,22}$ This precursor, obtained in a single step by ringopening of cheap and commercially available carbic anhydride, presented improved water solubility compared to native CS, which was attributed to the concomitant introduction of a carboxylate moiety acting as a solubilising group. In this current study, we investigate the physical properties of a series of CS-nb hydrogels using different thiol-based crosslinkers. We show that the structure and physicochemical properties of the hydrogels can be modulated by the choice of photo-crosslinker and assess their cytocompatibility and potential biomedical applicability.

For a minimum CS concentration of $1 \mathrm{w}: \mathrm{v} \%$, hydrogels employing CS-nb as the starting material were generated within seconds in all cases under UV-A, with Irgacure (IRG) as photoinitiator $^{21}$ and three different cross-linkers: a short and a long thiolated PEG derivative (namely $\mathrm{HS}-\mathrm{PEG}_{2}-\mathrm{SH}$ and $\mathrm{HS}-\mathrm{PEG}_{40}-\mathrm{SH}$ with respect to the number of ethylene glycol repeat units), and a thiolated CS (CS-SH), synthesized by ring-opening of Traut's reagent ${ }^{23}$, respectively (see Figure 1 for chemical structures). It was proposed that the final properties of 
CS-nb hydrogels would be influenced by the nature of the crosslinker structure. For instance, CS-SH presented multiple thiol functionalities closed to the CS backbone which are randomly located along the polymer chains, whereas both thiolated PEG derivatives are bifunctional and linear. The greater mobility provided by HS-PEG $40-\mathrm{SH}$ after crosslinking was anticipated to increase the hydrogels elasticity compared to the shorter variant $\mathrm{HS}_{-} \mathrm{PEG}_{2}-\mathrm{SH}$.

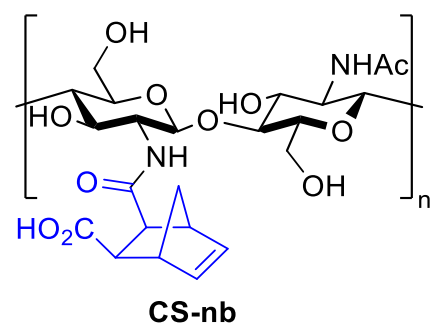

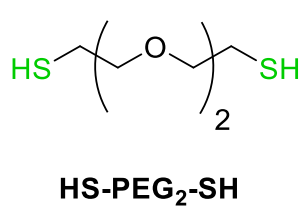

HS-PEG ${ }_{2}-\mathrm{SH}$

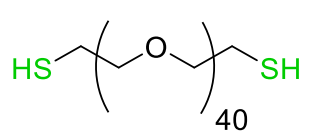

HS-PEG ${ }_{40}-\mathrm{SH}$

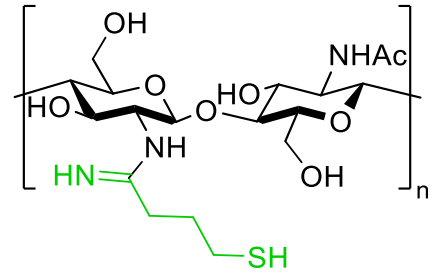

CS-SH

Figure 1. Chemical structures and abbreviations of the crosslinkers used in this work.

Next the physicochemical properties of the different hydrogels were evaluated. Interestingly, as a weak basic polyelectrolyte, CS hydrogels present both a $\mathrm{pH}$ - and saltresponsive swelling. ${ }^{24,25}$ For a fixed CS concentration $(2 \mathrm{w}: \mathrm{v} \%)$ and $[\mathrm{SH}]:[\mathrm{nb}]$ molar ratio $R_{S}$ $=1: 1$, the maximum swelling ratios $(\mathrm{SR})$ were obtained under acidic conditions $(\mathrm{SR} \sim 55)$ where amines are fully protonated and repel each other. Significant shrinkage was observed under basic conditions or in the presence of salts (SR 10-20), as expected from the neutralisation of the polymer chains (Table 1). Polyelectrolyte shrinkage generally occurs in the presence of salts due to ionic screening of the electrostatic interactions between polymer chains. ${ }^{26} \mathrm{CS}-\mathrm{SH}$ crosslinked hydrogels presented lower and less reproducible SR, possibly due to a more heterogeneous crosslinking. SR were slightly higher for hydrogels crosslinked with HS-PEG $40-\mathrm{SH}$ (SR 65), which is the longest and more flexible crosslinker, thus allowing for greater mobility between polymer chains and hence higher water uptake.

Table 1. Swelling ratios of $2 \%$ CS-nb hydrogels depending on the crosslinker.

\begin{tabular}{ccccc} 
& DI water & $\mathbf{p H}=\mathbf{3}$ & $\mathbf{p H}=\mathbf{1 1}$ & PBS \\
\hline HS-PEG $_{2}-\mathrm{SH}$ & $54.3 \pm 5.4$ & $64.4 \pm 11$ & $15.3 \pm 5.7$ & $12.9 \pm 0.43$ \\
HS-PEG $_{40}$-SH & $64.5 \pm 4.4$ & $54.5 \pm 4.8$ & $16.4 \pm 1.4$ & $19.3 \pm 0.79$ \\
CS-SH & $45.9 \pm 16.3$ & $54.9 \pm 17$ & $11.0 \pm 2.4$ & $9.0 \pm 0.77$
\end{tabular}


All hydrogels presented similar honeycomb-like structures with highly polydisperse pores of dimensions varying between $\sim 20$ and up to $\sim 120 \mu \mathrm{m}$, as evidenced by SEM (Figure 2). It is worth noting that SEM requires preliminary drying of the samples, which may affect the actual structure of the hydrogels. As anticipated, smaller pores were observed for HS-PEG $2-$ $\mathrm{SH}(d \sim 33 \pm 10 \mu \mathrm{m})$ than for HS-PEG 40 -SH-crosslinked materials $(d \sim 45 \pm 15 \mu \mathrm{m})$. Hydrogels employing CS-SH as crosslinker were more polydisperse in terms of pore dimensions, with both small pores of $d \sim 30 \mu \mathrm{m}$ but also more open geometries of dimensions $\sim 60-90 \mu \mathrm{m}$ observed.
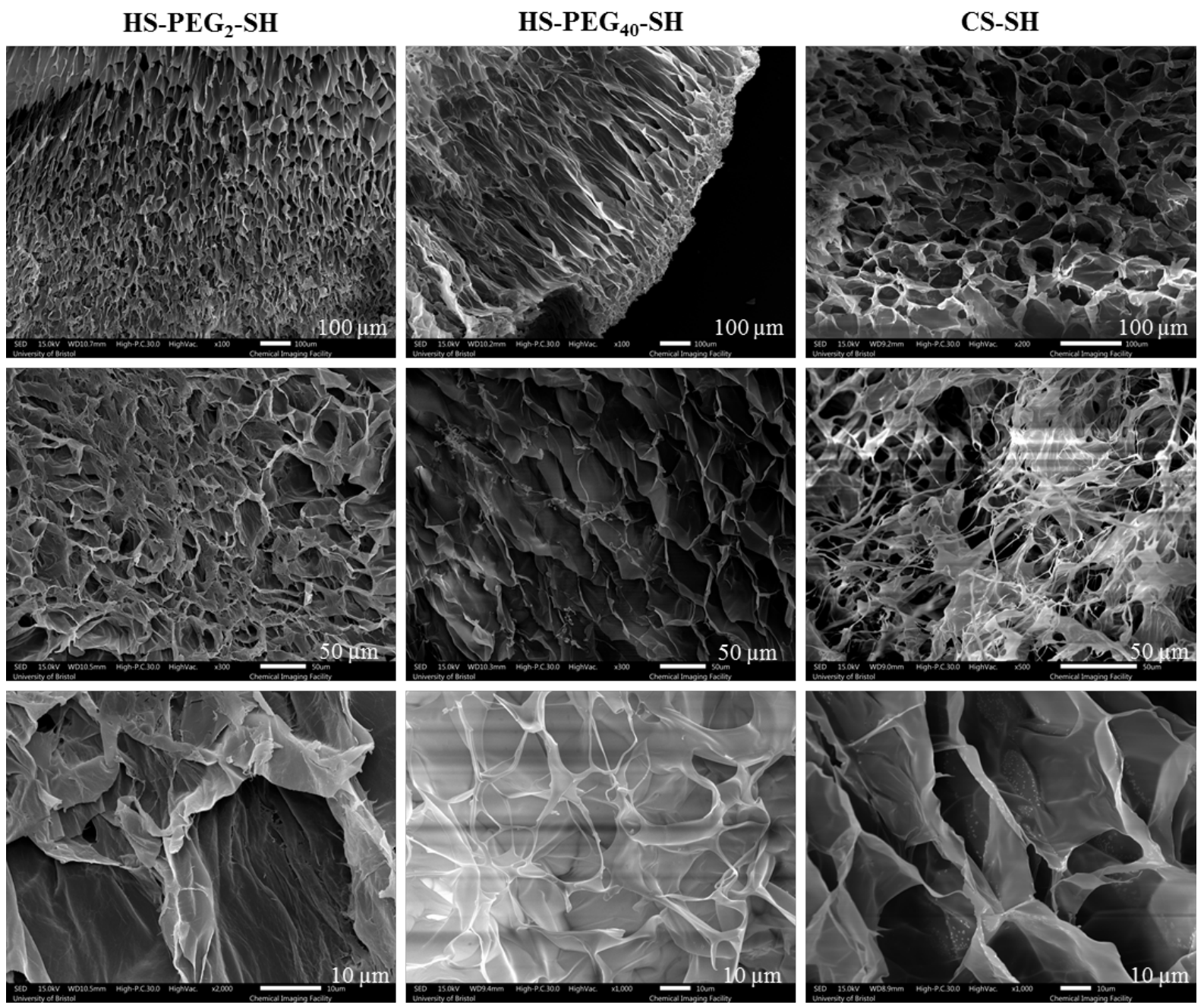

Figure 2. SEM images of CS-nb hydrogels crosslinked with: $\mathrm{HS}_{-} \mathrm{PEG}_{2}-\mathrm{SH}, \mathrm{HS}-\mathrm{PEG}_{40}-\mathrm{SH}$ and CS-SH.

All materials presented the rheological properties of viscoelastic covalent hydrogels: the storage modulus $G$ ' was at least 10 times higher than the loss modulus G', and all samples presented negligible frequency-dependency (Figure 3 and Figures S2-S5). The rheological properties of all hydrogels were highly dependent on the crosslinking density and the polymer 
concentration, with higher degree of crosslinking and polymer concentration yielding greater G' modulus (varying from $\sim 0.02$ to $\sim 20 \mathrm{kPa}$ ), which is consistent with previous reports. ${ }^{27-29}$ Hydrogels crosslinked with the short $\mathrm{HS}_{-} \mathrm{PEG}_{2}-\mathrm{SH}$ presented $\mathrm{G}^{\prime}$ modulus up to 10 times greater than materials crosslinked with $\mathrm{HS}_{-} \mathrm{PEG}_{40}-\mathrm{SH}$ or CS-SH. The crosslinker length directly impacts on how tightly the polymer chains are packed together in the gel, the shorter the distance between crosslinking points, the lower the chain mobility and therefore the greater the solid-like behaviour, as reflected by an increase in G'. HS-PEG $40-\mathrm{SH}$ is both longer and more flexible, allowing for more polymer chain rearrangement under shear. CS-SH should therefore present very tightly packed networks due to the high thiol density per polymer chain. The lower G' values suggest either incomplete crosslinking, possibly due to the random thiol localisation and their proximity to CS backbone which reduce their availability, or a more heterogeneous structure, consistent with the random thiol localisation along the chain.

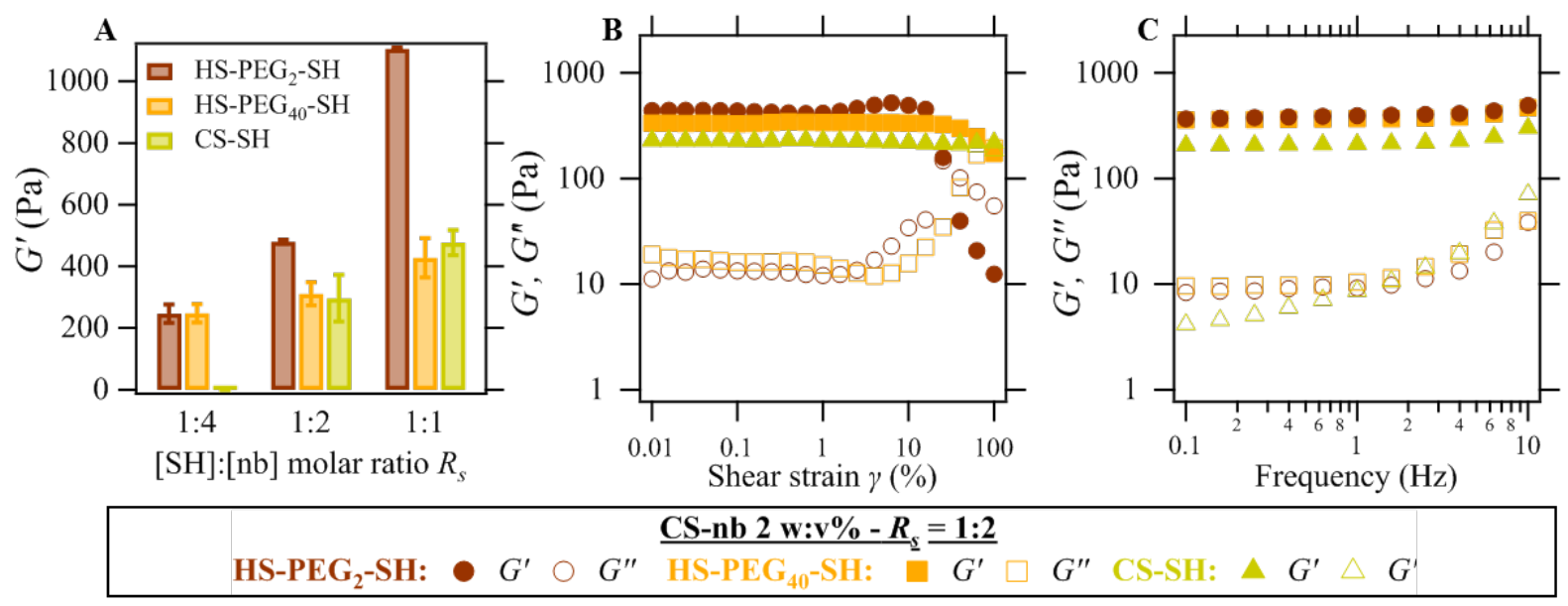

Figure 3. Rheology measurements of $2 \mathrm{w}: \mathrm{v} \%$ CS-nb hydrogels for different crosslinkers. (A) averaged $G^{\prime}$ and $G$ ', on the linear viscoelastic region determined by amplitude sweep measurements for various crosslinkers and $R_{s}$. (B) amplitude sweep and (C) frequency sweep measurements of $2 \% \mathrm{w}: \mathrm{v}$ CS-nb hydrogels crosslinked for $R_{S}=1: 2$.

The effect of the crosslinker on the internal structure of the hydrogels was further investigated using SANS. The formation of crosslinking points was evidenced by the linear increase in scattering intensity $I$ as $q$ decreased, related to the formation of large structures (Figure 4). This increase was characterized by a mass fractal of $\sim 2.4-2.6$ consistent with the formation of a crosslinked network (Table 2 and Table 3). The scattering of hydrogels is generally described by the combination of a liquid-like $\left(I_{L}(q)\right)$ and a solid-like $\left(I_{S}(q)\right)$ scattering terms modelling the polymer chain in solution and the crosslinking points respectively:

$$
I_{g e l}(q)=I_{L}(q)+I_{S}(q)
$$


The polymer contribution $I_{L}(q)$ is usually given by a Lorentzian equation:

$$
I_{L}(q)=\frac{I_{L}(0)}{1+\xi^{2} q^{2}}
$$

The solid-like contribution arises from heterogeneous crosslinking, leading to densely and poorly reticulated areas. ${ }^{31}$ These inhomogeneities are typically described by one of the following functions: another Gaussian term, an exponential function or with the DebyeBuesche-Anderson (DAB) model. ${ }^{31,32}$

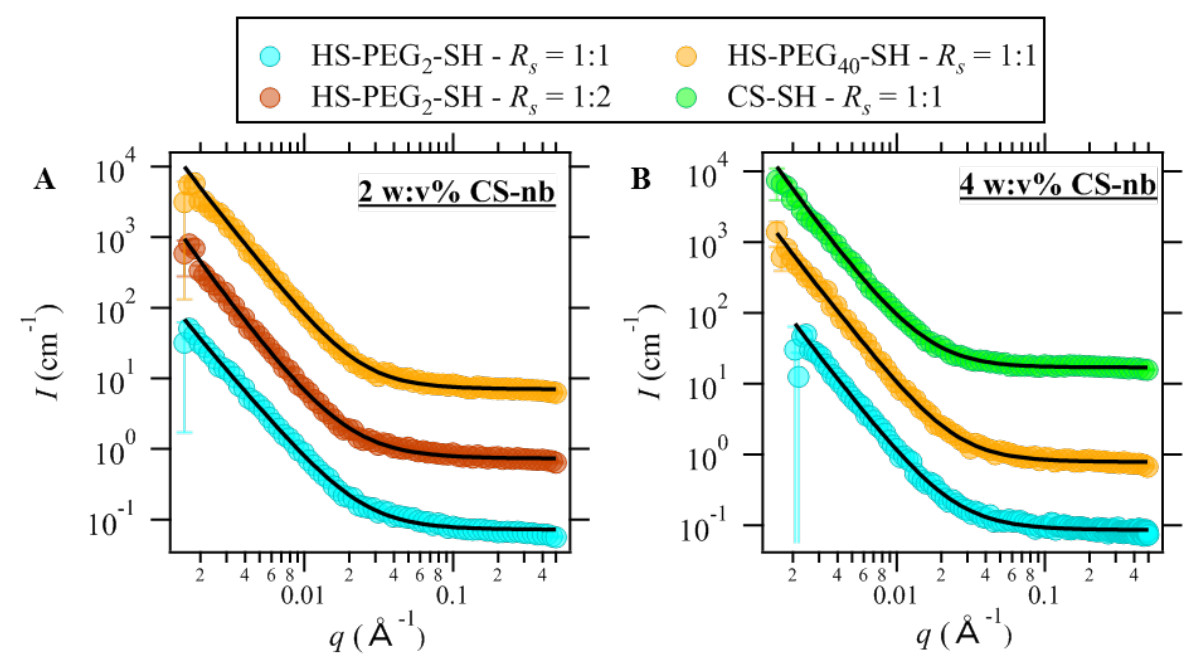

Figure 4. SANS data of CS-nb hydrogels. Fitted data are shown as a black line. Data have been offset (10x) on the $y$-axis for clarity.

Hammouda et al. investigated poly(ethylene oxide) (PEO) clustering mechanism leading to gel formation. The system was well modelled by the addition of a Lorentzian equation describing the polymer chain scattering and a Porod scattering term representing the clusters: ${ }^{33}$

$$
I(q)=\frac{A}{q^{D}}+\frac{C}{1+(q \xi)^{m}}+\text { background }
$$

where $D$ is the Porod exponent of the system, $m$ is the Lorentzian exponent, $\xi$ is the correlation length and A, C and the background are all constant. The Lorentzian exponent is related to the polymer-solvent interactions, with $m<2$ characteristics of efficient polymer solvation and $m$ $>2$ typically representative of polymer chains in a $\theta$ solvent. This model was successfully applied by Saffer et al. in their study of photo-chemically crosslinked PEG hydrogels through thiol-ene chemistry, a chemically similar system to ours. ${ }^{34}$ In addition, the introduction of the exponent $m$ on the Lorentzian term allows to account for hydrogen bond rich networks, frequent in polysaccharide-based hydrogels, for which a deviation on $I_{L}(q)$ has been observed. ${ }^{35}$ 
This equation described very well the scattering of the hydrogels. As the baseline was not flat the background scattering value B was fixed by fitting the high/intermediate $q$ region to a power law as described by Saffer et al.: ${ }^{34}$

$$
I(q)=\frac{A}{q^{D}}+B
$$

The mesh size obtained with Eq. 4 for $2 \mathrm{w}: \mathrm{v} \%$ hydrogels compares very well with those obtained by rheology, calculated with Eq. S4 (Table 2). As the degree of crosslinking increases from $R_{S}=1: 2$ to $1: 1$ the mesh size $\xi$ decreases from $\sim 29$ to $\sim 20 \mathrm{~nm}$, consistent with more crosslinking points bringing the polymer chains in closer proximity. The use of a longer crosslinker allows for bigger $\xi(19$ to $27 \mathrm{~nm})$ as expected. The Porod exponent, comprised between 2 and 3, is consistent with hydrogel formation, while the Lorentzian exponent is lower than 2 , indicating that the polymer chains are behaving as in a good solvent. ${ }^{34}$

The values of the mesh size obtained from SANS fitting for the $4 \mathrm{w}: \mathrm{v} \%$ hydrogels differ from those obtained by rheology, especially for $\mathrm{HS}_{-} \mathrm{PEG}_{2}-\mathrm{SH}$ and CS-SH hydrogels (Table 3). The general trend of $\xi$ increases from $\mathrm{HS}-\mathrm{PEG}_{2}-\mathrm{SH}$ to $\mathrm{HS}-\mathrm{PEG}_{40}-\mathrm{SH}$ is still verified. The smallest mesh size was measured for CS-SH hydrogels with SANS $(11 \mathrm{~nm})$ while its mechanical properties suggest a much bigger value $(17 \mathrm{~nm})$. Hyland et al. observed very different evolutions of rheological properties and $\xi$ obtained by neutrons, and suggested that the material mechanical properties was not only a result of the network density - measured as the correlation length $L_{c}$ using DAB model - but also of the fiber thickness and its packing. ${ }^{36}$ Rheology also investigates macroscopic properties, while SANS probes the polymer chain length scale; due to the high thiol content of CS-SH and its proximity to the polymer backbone, it is likely that these hydrogels were very heterogeneous, with highly reticulated area where the thiols could be accessed and others poorly crosslinked due to steric hindrance. The access to a smaller $q$ range would provide more information on the dimension of the resulting aggregates

Table 2. Summary of rheology and SANS characterisations of $2 \mathrm{w}: \mathrm{v} \%$ CS-nb hydrogels. Rheology: $\rho_{s}$ : crosslinking density, $\xi$ : mesh size, calculated from Eq. S3 and S4 respectively. SANS: $D$ : fractal dimension, $m$ : Lorentzian exponent in Eq. 3.

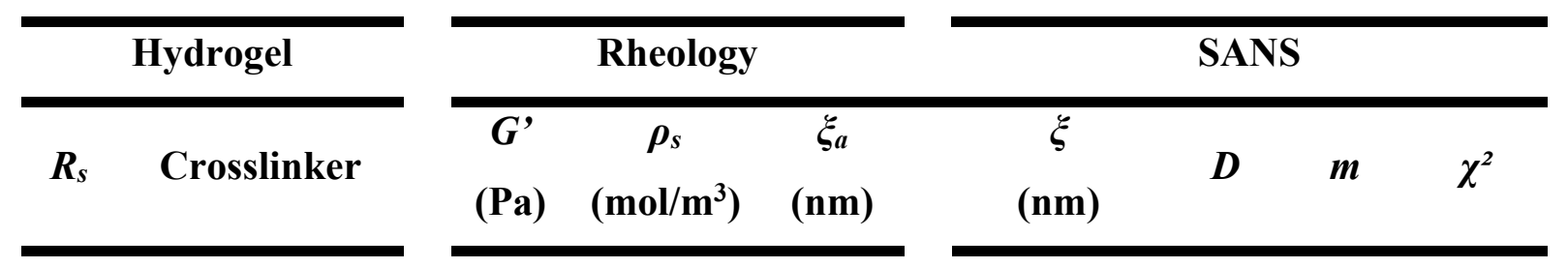




\begin{tabular}{|c|c|c|c|c|c|c|c|c|}
\hline $1: 1$ & HS-PEG $2-S H$ & 1107 & 1.17 & 19.2 & $18.4 \pm 3.5$ & 2.43 & 1.43 & 3.65 \\
\hline $1: 2$ & HS-PEG $2-S H$ & 479 & 0.19 & 25.5 & $29.3 \pm 2.3$ & 2.79 & 1.58 & 3.98 \\
\hline $1: 1$ & HS-PEG $40-\mathrm{SH}$ & 435 & 0.18 & 26.2 & $27.9 \pm 4.9$ & 2.60 & 1.37 & 3.59 \\
\hline $1: 1$ & CS-SH & 477 & 0.19 & 25.5 & _ & - & - & - \\
\hline
\end{tabular}

Table 3. Summary of rheology and SANS characterisations of $4 \mathrm{w}: \mathrm{v} \%$ CS-nb hydrogels. Rheology: $\rho_{s}$ : crosslinking density, $\xi$ : mesh size, calculated from Eq. S3 and S4 respectively. SANS: $D$ : fractal dimension, $m$ : Lorentzian exponent in Eq. 3.

\begin{tabular}{|c|c|c|c|c|c|c|c|c|}
\hline \multicolumn{2}{|r|}{ Hydrogel } & \multicolumn{3}{|c|}{ Rheology } & \multicolumn{4}{|c|}{ SANS } \\
\hline $\mathbf{R}_{\mathrm{s}}$ & Crosslinker & $\begin{array}{c}\mathbf{G}^{\prime} \\
(\mathbf{P a})\end{array}$ & $\begin{array}{c}\rho_{\mathrm{s}} \\
\left(\mathrm{mol} / \mathrm{m}^{3}\right)\end{array}$ & $\begin{array}{c}\xi_{\mathrm{a}} \\
(\mathrm{nm})\end{array}$ & $\begin{array}{c}\xi \\
(\mathrm{nm})\end{array}$ & D & $\mathbf{m}$ & $\chi^{2}$ \\
\hline $1: 1$ & $\mathrm{HS}^{-\mathrm{PEG}_{2}-\mathrm{SH}}$ & 11560 & 4.67 & 8.79 & $15.2 \pm 0.3$ & 2.61 & 1.43 & 1.49 \\
\hline $1: 1$ & HS-PEG $40-\mathrm{SH}$ & 1695 & 0.67 & 16.8 & $20.0 \pm 4.7$ & 2.60 & 1.42 & 1.83 \\
\hline $1: 1$ & $\mathrm{CS}-\mathrm{SH}$ & 1535 & 0.67 & 17.2 & $11.3 \pm 0.7$ & 2.73 & 1.95 & 4.37 \\
\hline
\end{tabular}

The fractal dimension $D$ is also relevant to biological studies, as shown by Hung et al. on agarose physical hydrogels. They synthesized a variety of hydrogels of matching rheological properties but different mass fractal, which they measured both by rheology and with SAXS. While the impact of rheology on cell culture is well-known, they were the first to observe that hydrogel fractal dimension also had a significant impact on stem cell differentiation. ${ }^{37} \mathrm{CS}-\mathrm{nb}$ hydrogels studied with SANS presented very similar mass fractals $D \sim 2.6$ suggesting that cell toxicity assays may be directly correlated to the hydrogel rheological properties. In addition, the range of achieved mechanical properties $(0.020-20 \mathrm{kPa})$ was relevant to a wide area of biomedical applications, ranging from neural tissue engineering $(\sim 100 \mathrm{~Pa})$ to muscle or skin cell culture $(\sim 10 \mathrm{kPa}){ }^{38}$

Hydrogels of tailored shapes could be easily obtained using moulds (Error! Reference source not found.A). To demonstrate the ability of our hydrogels to encapsulate and slowly release molecules overtime, fluorescent dye rhodamine B was used as a model compound and entrapped within the hydrogel structure during the gelation process. After one week of immersion in water, the gel had swollen and lost most of its colour due to rhodamine release (Error! Reference source not found.A). Although the rate of release was not quantified, this highlights the possible application of these hydrogels for drug delivery, especially due to their 
improved swelling properties under acidic conditions, which is a common feature of tumours and infected organs, and is currently under studies.

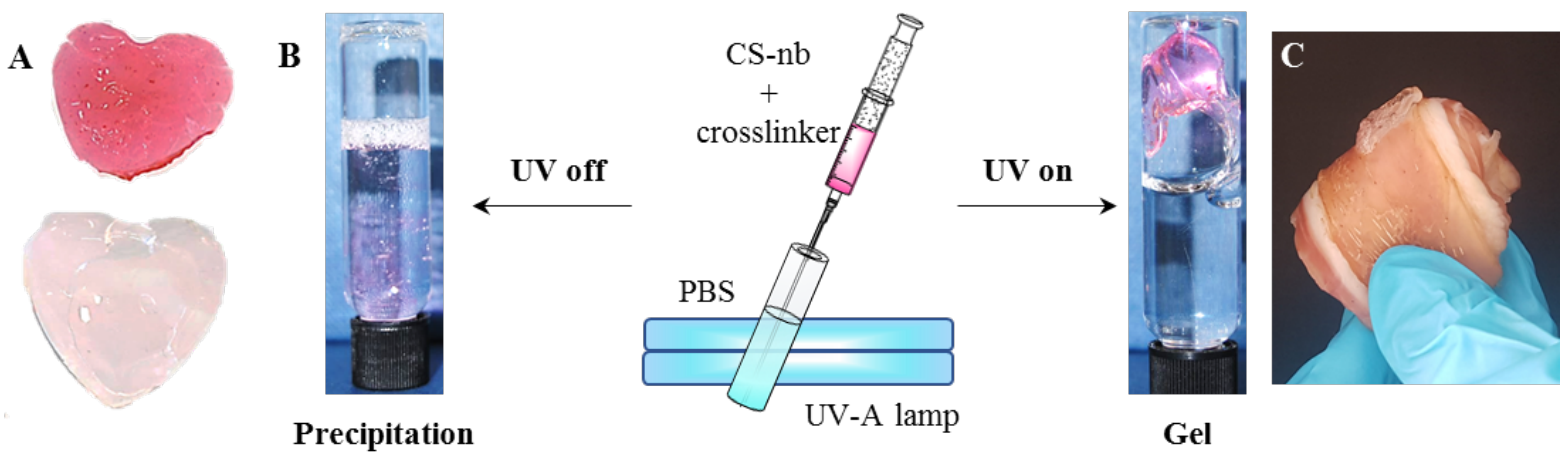

Figure 5. Potential CS-nb hydrogel applications. (A) Drug-release: shape-retaining heart with encapsulated rhodamine as generated (top) and after one week of storage in water (bottom). (B) In-situ generated hydrogels in PBS. (C) Hydrogel adhesion on pork skin (in situsynthesised) Owing to the very fast gelation kinetics, hydrogels could be generated in situ when the liquid polymer precursor was injected in PBS under UV, independently of the crosslinker used $\left(R_{s}=1: 1\right)$. Important to note that if the UV source was switched off, CS precipitation occurred upon injection due to its low solubility under physiological conditions. (Figure 5B). Hydrogels could also be generated in situ directly on pork skin, both in dry state and in PBS, and gel adhesion was observed on the skin that was stored in PBS for up to 4 days (Error! Reference source not found.B,C) suggesting potential applications in wound healing. The adhesion is most likely due to the generation of free radicals in the initiation step which can also recombine with functional groups present on the skin. ${ }^{39,}{ }^{40} \mathrm{CS}-\mathrm{nb}$ precursor solutions containing IRG could also be sterilised in an autoclave before triggering the gelation with UV, since no structural change were observed on the precursors solution by ${ }^{1} \mathrm{H}$ NMR.

Hydrogels have been widely studied for cell culture as they provide a 3D structure for cells to grow and develop which is highly similar to human tissues. ${ }^{41}$ Substrates favouring skin wound healing and fibroblast growth frequently present $\mathrm{G}^{\prime} \sim 0.5-10 \mathrm{kPa},{ }^{42-44}$ which informed the choice of hydrogels for initial toxicity testing ( 3 crosslinkers at either 2 or $4 \mathrm{w}: \mathrm{v} \%, R_{s}=$ 1:1), which covers a wide range of rheology and swelling properties. A good hydrogel candidate for a tissue engineering approach must allow cells to adhere, infiltrate and spread in the scaffold; failing to do this will lead to cell toxicity, which can be assessed by metabolic activity measurements or live cell fluorescent staining. Human skin fibroblasts (HDF), a standard model for wound healing and tissue engineering applications, and placenta choriocarcinoma cells (BeWo-b33), an established model of the placental membrane ${ }^{45}$ and relevant to wound healing as an epithelial monolayer, were cultured on the six different chosen 
hydrogels. Both cell lines presented limited adhesion on the gel substrate, with better spreading for BeWo on softer gels ( $2 \mathrm{w}: \mathrm{v} \%$ ) and for HDF on stiffer gels (4 w:v\%), while spheroids where observed on the softest substrates, indicating unsuccessful adhesion and consequent cell death (Figure 5). The metabolic activity as measured using Alamar Blue (AB) reagent was between 60 and $80 \%$ for BeWo compared to $\sim 20-35 \%$ for HDF on the softest substrates ( $2 \mathrm{w}: \mathrm{v} \%)$, which increased up to $80 \%$ on the $4 \mathrm{w}: \mathrm{v} \%$ hydrogels (Figure 5). The observed stiffnessspreading relationship of HDF is consistent with previous work on non-biofunctionalised acrylate hydrogels - i.e. without the introduction of peptides or sugars to impact on adhesion. ${ }^{46,}$ 47
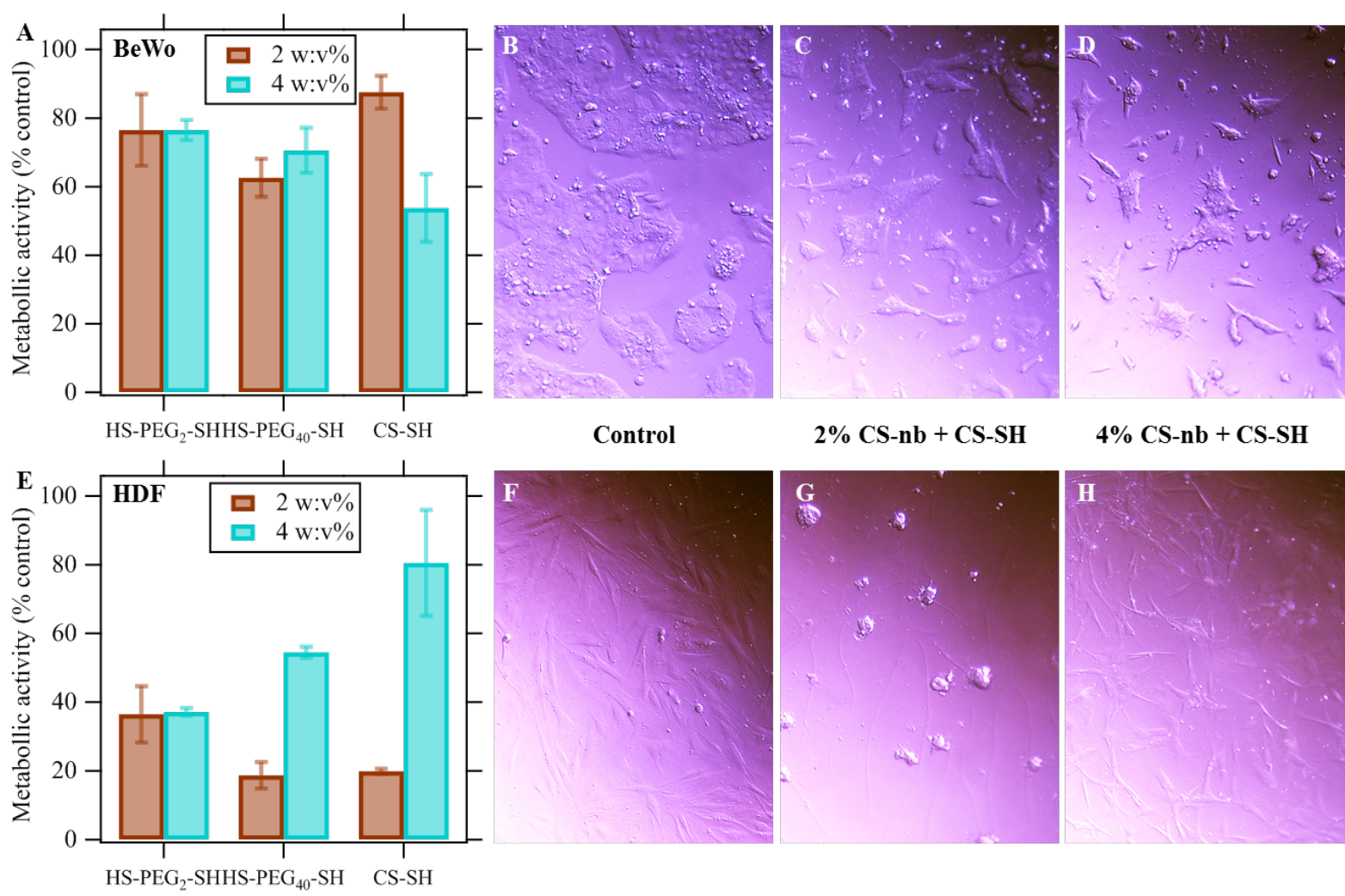

Control

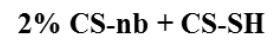

$4 \%$ CS-nb + CS-SH
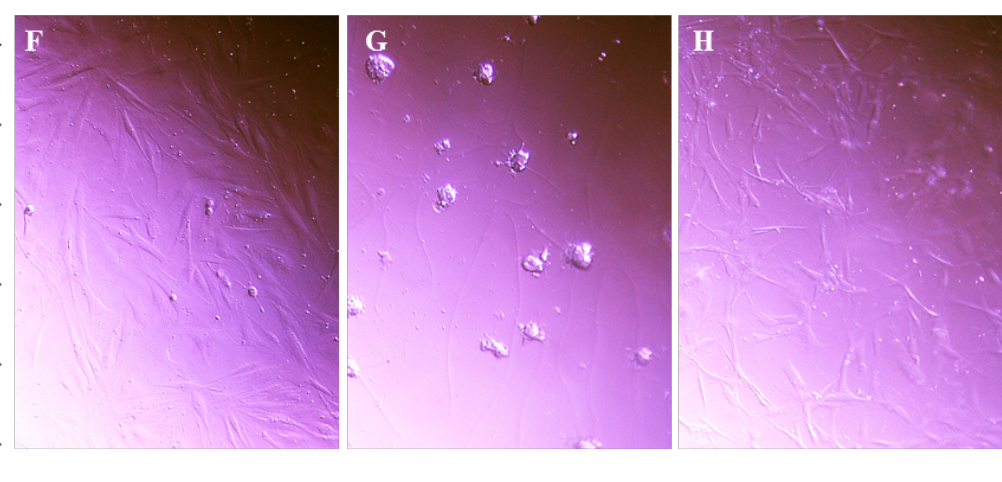

Figure 5. Cell viability relative to controls $(100 \%)(A, E)$ and widefield microscopy images of BeWo (B-D) and HDF (F-H) cells 24 hrs after seeding on: the plate (B, F), CS-SH crosslinked $2 \mathrm{w}: \mathrm{v} \%$ hydrogels $(\mathrm{C}, \mathrm{G})$ and CS-SH crosslinked $2 \mathrm{w}: \mathrm{v} \%$ hydrogels with 0.25 $\mathrm{w}: \mathrm{v} \%$ collagen.

Although CS presents similarities with GAG in the ECM, its structure often fails to promote cell adhesion ${ }^{48}$ and functionalisation with adhesion peptides such as $\mathrm{RGD}^{49}$ hyaluronic acid, ${ }^{50}$ or proteins e.g. collagen $(\mathrm{Col})^{51}$ or gelatin ${ }^{52}$ is common. The incorporation of Col (type I) into CS hydrogels (final concentration CS $2 \mathrm{w}: \mathrm{v} \%$ - Col $0.25 \mathrm{w}: \mathrm{v} \%$ ) drastically improved HDF spreading and adhesion, resulting in viabilities greater than $90 \%$ for up to 6 
days, while having minimum effect on BeWo (Figure6). It is worth noting that only a small amount of Col was needed to significantly enhance cell adhesion, while Col hydrogels typically require concentrations higher than $0.5 \mathrm{w}: \mathrm{v} \%$ to achieve good viability ${ }^{53}$ Lower viabilities were generally observed when CS-SH was used as a crosslinker (Figure 6). The incorporation of Col into CS hydrogels had different impact on their rheological properties. While HS-PEG40$\mathrm{SH}$ crosslinked hydrogels presented similar $\mathrm{G}$ ' values with and without $\mathrm{Col}, \mathrm{HS}-\mathrm{PEG}_{2}-\mathrm{SH}$ substrates were slightly stiffer $\left(\mathrm{G}^{\prime} \sim 1\right.$ to $\left.\sim 2 \mathrm{kPa}\right)$ and CS-SH materials were drastically weaker $\left(\mathrm{G}^{\prime} \sim 500\right.$ to $\left.\sim 80 \mathrm{~Pa}\right)$. SEM revealed that PEG crosslinked CS/Col hydrogels retained their honeycomb structure, with the presence of large, elongated and regularly spaced pores for HS$\mathrm{PEG}_{2}-\mathrm{SH}$ hydrogels and spherical ones for HS-PEG $40-\mathrm{SH}$ materials. CS-SH crosslinked hybrids, however, presented a highly polydisperse structure, with the presence of numerous pores of smaller dimensions and random directions, as well as fibre-like regions, suggesting poor mixing between CS and Col (Figure S7). The interaction between Col and CS may prevent the formation of some crosslinks, especially with CS-SH where the thiol groups are very closed to the polymer backbone, and result in the smaller $G$ ' values obtained. In addition, interactions between charged groups of both polymers will occur, which may reduce the crosslinking efficiency by hindering the nb moieties or increase network inhomogeneity. These structural observations agree broadly with the rheology results, in which a loss in structural organisation related to poorer mechanical properties is noted. This also correlates with the observed cell viability, where both the presence of the RGD binding domains of Col and a significant stiffness are required to achieve efficient adhesion of HDF and good viabilities. 

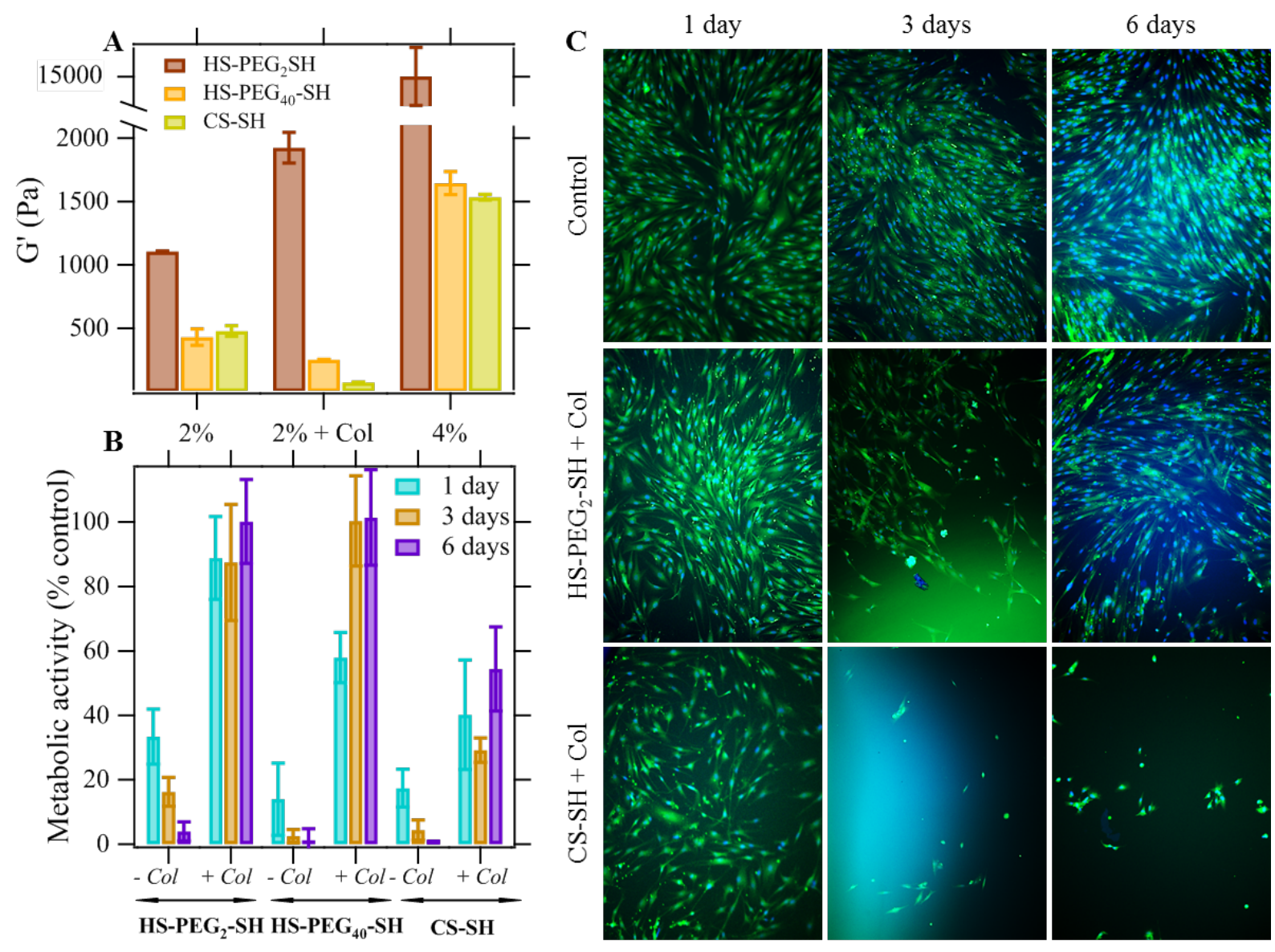

Figure 6. Characterisation of hydrogels investigated for cell studies. Rheological properties of all hydrogels cultured with HDF (A), metabolic activity (B) and widefield microscopy imaging of (C) cells after 1, 3 and 6 days of culture on CS hydrogels crosslinked with HS-PEG $2-\mathrm{SH}$ or $\mathrm{CS}-\mathrm{SH}$, with or without $0.25 \mathrm{w}: \mathrm{v} \% \mathrm{Col}$, compared to cells grown on plates (control).

In conclusion, a series of photo-crosslinked CS-based hydrogels were successfully synthesized by thiol-ene click reaction from a simple CS-nb precursor and different crosslinkers under UV exposure. The material properties could be readily tuned at the macroand microscale by changing the crosslinking density, the nature of the crosslinker and the polymer concentration. The pore size dimensions, ranging between $20-100 \mu \mathrm{m}$ and the rheological properties tuneable around $0.5-20 \mathrm{kPa}$ were compatible with cells growth after addition of a low concentration of Col. Due to the fast gelation kinetics, hydrogels could be successfully generated in situ under dry and wet conditions and adhered to pork skin, could be molded into stable shapes and could release entrapped compounds. All these conditions make CS-nb a potential candidate for biomedical applications in areas such as wound healing or tissue engineering. 


\section{Associated content}

\section{Supporting Information}

The Supporting Information is available free of charge on the ACS Publications website at DOI: .... Detailed experimental procedures and characterization data (PDF)

\section{Author information}

\section{Corresponding Authors}

*E-mail: m.c.galan@bristol.ac.uk. Phone: +44 (0) 117-928- 7654.

*E-mail: wuge.briscoe@bristol.ac.uk; Phone: +44 (0)1173318256

\section{Author Contributions}

S.M. performed all the experiments and data analysis. S.R. provided assistance on the SANS beamline. The manuscript was written through contributions of all authors, who also contributed to the data analysis and interpretation. All authors have given approval to the final version of the manuscript.

\section{Notes}

The authors declare no competing financial interest.

\section{Acknowledgments}

We thank Professor Craig Butts for helpful discussions with NMRs, Paul Lehman for help with the NMR, Dr Margaret Saunders for providing the BeWo-b33 cells, Dr Jean-Charles Eloi for help and discussion with SEM. MCG and SM were supported by the European Research Council (ERC-COG: 648239) and the Bristol Chemical Synthesis Centre for Doctoral Training (Engineering and Physical Science Research Council (EPSRC) EP/L015366/1). This publication is based upon work from COST Action GLYCONanoPROBES (CA18132), supported by COST (European Cooperation in Science and Technology).

\section{References}

1. Zhu, J.; Marchant, R. E., Design properties of hydrogel tissue-engineering scaffolds. Expert Review of Medical Devices 2011, 8 (5), 607-626.

2. Guo, J. L.; Kim, Y. S.; Mikos, A. G., Biomacromolecules for Tissue Engineering: Emerging Biomimetic Strategies. Biomacromolecules 2019.

3. Guan, X.; Avci-Adali, M.; Alarçin, E.; Cheng, H.; Kashaf, S. S.; Li, Y.; Chawla, A.; Jang, H. L.; Khademhosseini, A., Development of hydrogels for regenerative engineering. Biotechnology Journal 2017, 1600394-n/a. 
4. Radhakrishnan, J.; Subramanian, A.; Krishnan, U. M.; Sethuraman, S., Injectable and 3D Bioprinted Polysaccharide Hydrogels: From Cartilage to Osteochondral Tissue Engineering. Biomacromolecules 2017, 18 (1), 1-26.

5. Khan, F.; Ahmad, S. R., Polysaccharides and Their Derivatives for Versatile Tissue Engineering Application. Macromolecular Bioscience 2013, 13 (4), 395-421.

6. Dragan, E. S.; Dinu, M. V., Polysaccharides constructed hydrogels as vehicles for proteins and peptides. A review. Carbohydrate Polymers 2019, 225, 115210.

7. Kasaai, M. R., Determination of the degree of $\mathrm{N}$-acetylation for chitin and chitosan by various NMR spectroscopy techniques: A review. Carbohydrate Polymers 2010, 79 (4), 801810.

8. Croisier, F.; Jérôme, C., Chitosan-based biomaterials for tissue engineering. European Polymer Journal 2013, 49 (4), 780-792.

9. Bernkop-Schnürch, A.; Dünnhaupt, S., Chitosan-based drug delivery systems. European Journal of Pharmaceutics and Biopharmaceutics 2012, 81 (3), 463-469.

10. Bhattarai, N.; Gunn, J.; Zhang, M., Chitosan-based hydrogels for controlled, localized drug delivery. Adv Drug Deliv Rev 2010, 62 (1), 83-99.

11. Sahariah, P.; Másson, M., Antimicrobial Chitosan and Chitosan Derivatives: A Review of the Structure-Activity Relationship. Biomacromolecules 2017, 18 (11), 3846-3868.

12. Saporito, F.; Sandri, G.; Rossi, S.; Bonferoni, M. C.; Riva, F.; Malavasi, L.; Caramella, C.; Ferrari, F., Freeze dried chitosan acetate dressings with glycosaminoglycans and traxenamic acid. Carbohydrate Polymers 2018, 184, 408-417.

13. Tiwari, S.; Patil, R.; Bahadur, P., Polysaccharide Based Scaffolds for Soft Tissue Engineering Applications. 2019, 11 (1), 1.

14. Boecker, A.; Daeschler, S. C.; Kneser, U.; Harhaus, L., Relevance and Recent Developments of Chitosan in Peripheral Nerve Surgery. 2019, 13 (104).

15. Pei, M.; Mao, J.; Xu, W.; Zhou, Y.; Xiao, P., Photocrosslinkable chitosan hydrogels and their biomedical applications. J. Polym. Sci. Part A: Polym. Chem. 2019, (doi:10.1002/pola.29305), 41563-41574.

16. Fairbanks, B. D.; Love, D. M.; Bowman, C. N., Efficient Polymer-Polymer Conjugation via Thiol-ene Click Reaction. Macromolecular Chemistry and Physics 2017, 218 (18), 1700073-n/a.

17. Hoyle, C. E.; Lee, T. Y.; Roper, T., Thiol-enes: Chemistry of the past with promise for the future. 2004, 42 (21), 5301-5338.

18. Wang, Z.; Jin, X.; Dai, R.; Holzman, J. F.; Kim, K., An ultrafast hydrogel photocrosslinking method for direct laser bioprinting. RSC Advances 2016, 6 (25), 2109921104.

19. Shih, H.; Lin, C.-C., Visible-Light-Mediated Thiol-Ene Hydrogelation Using Eosin-Y as the Only Photoinitiator. Macromolecular Rapid Communications 2013, 34 (3), 269-273.

20. Lee, H. J.; Fernandes-Cunha, G. M.; Myung, D., In situ-forming hyaluronic acid hydrogel through visible light-induced thiol-ene reaction. Reactive and Functional Polymers 2018, 131, 29-35.

21. Michel, S. E. S.; Dutertre, F.; Denbow, M. L.; Galan, M. C.; Briscoe, W. H., Facile Synthesis of Chitosan-Based Hydrogels and Microgels through Thiol-Ene Photoclick CrossLinking. ACS Applied Bio Materials 2019, 2 (8), 3257-3268.

22. Michel, S. S. E.; Kilner, A.; Eloi, J.-C.; Rogers, S. E.; Briscoe, W. H.; Galan, M. C., Norbornene-Functionalized Chitosan Hydrogels and Microgels via Unprecedented Photoinitiated Self-Assembly for Potential Biomedical Applications. ACS Applied Bio Materials 2020, 3 (8), 5253-5262. 
23. Bernkop-Schnürch, A.; Hornof, M.; Zoidl, T., Thiolated polymers - thiomers: synthesis and in vitro evaluation of chitosan-2-iminothiolane conjugates. International Journal of Pharmaceutics 2003, 260 (2), 229-237.

24. Qu, X.; Wirsén, A.; Albertsson, A. C., Novel pH-sensitive chitosan hydrogels: swelling behavior and states of water. Polymer 2000, 41 (12), 4589-4598.

25. Zhu, L.; Bratlie, K. M., pH sensitive methacrylated chitosan hydrogels with tunable physical and chemical properties. Biochemical Engineering Journal 2018, 132, 38-46.

26. Chang, C.; He, M.; Zhou, J.; Zhang, L., Swelling Behaviors of pH- and SaltResponsive Cellulose-Based Hydrogels. Macromolecules 2011, 44 (6), 1642-1648.

27. Gramlich, W. M.; Kim, I. L.; Burdick, J. A., Synthesis and orthogonal photopatterning of hyaluronic acid hydrogels with thiol-norbornene chemistry. Biomaterials 2013, 34 (38), 9803-11.

28. Lee, S.; Park, Y. H.; Ki, C. S., Fabrication of PEG-carboxymethylcellulose hydrogel by thiol-norbornene photo-click chemistry. Int J Biol Macromol 2016, 83, 1-8.

29. McOscar, T. V. C.; Gramlich, W. M. J. C., Hydrogels from norbornene-functionalized carboxymethyl cellulose using a UV-initiated thiol-ene click reaction. 2018, 25 (11), 65316545 .

30. Munoz, Z.; Shih, H.; Lin, C.-C., Gelatin hydrogels formed by orthogonal thiolnorbornene photochemistry for cell encapsulation. Biomaterials Science 2014, 2 (8), 1063 1072.

31. Gomes, C.; Dias, R. C. S.; Costa, M. R. P. F. N., Static Light Scattering Monitoring and Kinetic Modeling of Polyacrylamide Hydrogel Synthesis. 2019, 7 (4), 237.

32. Chalal, M.; Ehrburger-Dolle, F.; Morfin, I.; Bley, F.; Aguilar de Armas, M.-R.; López Donaire, M.-L.; San Roman, J.; Bölgen, N.; Pişkin, E.; Ziane, O.; Casalegno, R., SAXS Investigation of the Effect of Temperature on the Multiscale Structure of a Macroporous Poly(N-isopropylacrylamide) Gel. Macromolecules 2010, 43 (4), 2009-2017.

33. Hammouda, B.; Ho, D. L.; Kline, S., Insight into Clustering in Poly(ethylene oxide) Solutions. Macromolecules 2004, 37 (18), 6932-6937.

34. Saffer, E. M.; Lackey, M. A.; Griffin, D. M.; Kishore, S.; Tew, G. N.; Bhatia, S. R., SANS study of highly resilient poly(ethylene glycol) hydrogels. Soft Matter 2014, 10 (12), 1905-1916.

35. Horkay, F.; Hecht, A. M.; Mallam, S.; Geissler, E.; Rennie, A. R., Macroscopic and microscopic thermodynamic observations in swollen poly(vinyl acetate) networks. Macromolecules 1991, 24 (10), 2896-2902.

36. Hyland, L. L.; Taraban, M. B.; Feng, Y.; Hammouda, B.; Yu, Y. B., Viscoelastic properties and nanoscale structures of composite oligopeptide-polysaccharide hydrogels. Biopolymers 2012, 97 (3), 177-88.

37. Hung, K.-C.; Jeng, U. S.; Hsu, S.-h., Fractal Structure of Hydrogels Modulates Stem Cell Behavior. ACS Macro Letters 2015, 4 (9), 1056-1061.

38. Li, X.; Katsanevakis, E.; Liu, X.; Zhang, N.; Wen, X., Engineering neural stem cell fates with hydrogel design for central nervous system regeneration. Progress in Polymer Science 2012, 37 (8), 1105-1129.

39. Ghobril, C.; Grinstaff, M. W., The chemistry and engineering of polymeric hydrogel adhesives for wound closure: a tutorial. Chemical Society Reviews 2015, 44 (7), 1820-1835.

40. Zavada, S. R.; McHardy, N. R.; Scott, T. F., Oxygen-Mediated Enzymatic Polymerization of Thiol-Ene Hydrogels. J Mater Chem B 2014, 2 (17), 2598-2605.

41. Gupta, A.; Kowalczuk, M.; Heaselgrave, W.; Britland, S. T.; Martin, C.; Radecka, I., The production and application of hydrogels for wound management: A review. European Polymer Journal 2019, 111, 134-151. 
42. Zhou, Y.; Zhao, S.; Zhang, C.; Liang, K.; Li, J.; Yang, H.; Gu, S.; Bai, Z.; Ye, D.; $\mathrm{Xu}, \mathrm{W}$., Photopolymerized maleilated chitosan/thiol-terminated poly (vinyl alcohol) hydrogels as potential tissue engineering scaffolds. Carbohydrate Polymers 2018, 184, 383-389.

43. Moore, A. L.; Marshall, C. D.; Barnes, L. A.; Murphy, M. P.; Ransom, R. C.; Longaker, M. T., Scarless wound healing: Transitioning from fetal research to regenerative healing. 2018, 7 (2), e309.

44. Achterberg, V. F.; Buscemi, L.; Diekmann, H.; Smith-Clerc, J.; Schwengler, H.; Meister, J.-J.; Wenck, H.; Gallinat, S.; Hinz, B., The Nano-Scale Mechanical Properties of the Extracellular Matrix Regulate Dermal Fibroblast Function. Journal of Investigative Dermatology 2014, 134 (7), 1862-1872.

45. Correia Carreira, S.; Cartwright, L.; Mathiesen, L.; Knudsen, L. E.; Saunders, M., Studying placental transfer of highly purified non-dioxin-like PCBs in two models of the placental barrier. Placenta 2011, 32 (3), 283-291.

46. Çolak, A.; Li, B.; Blass, J.; Koynov, K.; del Campo, A.; Bennewitz, R., The mechanics of single cross-links which mediate cell attachment at a hydrogel surface. Nanoscale 2019, 11 (24), 11596-11604.

47. Hersel, U.; Dahmen, C.; Kessler, H., RGD modified polymers: biomaterials for stimulated cell adhesion and beyond. Biomaterials 2003, 24 (24), 4385-4415.

48. Jiang, Y.; Deng, Y.; Tu, Y.; Ay, B.; Sun, X.; Li, Y.; Wang, X.; Chen, X.; Zhang, L., Chitosan-based asymmetric topological membranes with cell-like features for healthcare applications. Journal of Materials Chemistry B 2019, 7 (16), 2634-2642.

49. Park, K. M.; Joung, Y. K.; Park, K. D.; Lee, S. Y.; Lee, M. C. J. M. R., RGDConjugated chitosan-pluronic hydrogels as a cell supported scaffold for articular cartilage regeneration. 2008, 16 (6), 517-523.

50. Deng, Y.; Ren, J.; Chen, G.; Li, G.; Wu, X.; Wang, G.; Gu, G.; Li, J., Injectable in situ cross-linking chitosan-hyaluronic acid based hydrogels for abdominal tissue regeneration. Scientific Reports 2017, 7 (1), 2699.

51. Reyna-Urrutia, V. A.; Mata-Haro, V.; Cauich-Rodriguez, J. V.; Herrera-Kao, W. A.; Cervantes-Uc, J. M., Effect of two crosslinking methods on the physicochemical and biological properties of the collagen-chitosan scaffolds. European Polymer Journal 2019, 117, 424-433. 52. da Silva, M. A.; Bode, F.; Drake, A. F.; Goldoni, S.; Stevens, M. M.; Dreiss, C. A., Enzymatically Cross-Linked Gelatin/Chitosan Hydrogels: Tuning Gel Properties and Cellular Response. 2014, 14 (6), 817-830.

53. Yang, H.; Shen, L.; Bu, H.; Li, G., Stable and biocompatible hydrogel composites based on collagen and dialdehyde carboxymethyl cellulose in a biphasic solvent system. Carbohydrate Polymers 2019, 222, 114974. 